\title{
NONLINEAR RADIATIVE HEAT TRANSFER TO CARREAU FLUID OVER A NONLINEAR STRETCHING SHEET IN A POROUS MEDIUM IN THE PRESENCE OF NON-UNIFORM HEAT SOURCE/SINK AND VISCOUS DISSIPATION
}

\author{
M. Umeshaiah ${ }^{1}$, M. R. Krishnamurthy ${ }^{2}$, N.G. Rudraswamy ${ }^{3}$, B. J. Gireesha ${ }^{4}$ and B.C. Prasannakumara ${ }^{5, *}$ \\ ${ }^{1}$ Department of Mathematics, PESITM College of Engineering, Shimoga-577204, Karnataka, India \\ ${ }^{2}$ Department of Mathematics, Jawaharlal Nehru College of Engineering, Shimoga-577204, Karnataka, India \\ ${ }^{3}$ Department of Mathematics, Sahyadri Science College, Shimoga-577201, Karnataka, India \\ ${ }^{4}$ Department of Studies and Research in Mathematics, Kuvempu University, Shankaraghatta-577 451, Shimoga, Karnataka, India \\ ${ }^{5}$ Department of Mathematics, Government First Grade College, Koppa, Chikkamagaluru-577126, Karnataka, Inida.
}

\begin{abstract}
This article presents the effect of nonlinear thermal radiation on boundary layer flow and heat transfer of Carreau fluid model over a nonlinear stretching sheet embedded in a porous medium in the presence of non-uniform heat source/sink and viscous dissipation with convective boundary condition. The governing partial differential equations with the corresponding boundary conditions are reduced to a set of ordinary differential equations using similarity transformation, which is then solved numerically by the fourth-fifth order Runge-Kutta-Fehlberg integration scheme featuring a shooting technique. The influence of significant parameters such as power law index parameter, Stretching parameter, Weissenberg number, permeability parameter, temperature ratio parameter, radiation parameter, Biot number, heat source/sink parameters, Eckert number and Prandtl number on the flow and heat transfer characteristics is discussed. The obtained results shows that for shear thinning fluid the fluid velocity is depressed by the Weissenberg number while opposite behavior for the shear thickening fluid is observed. A comparison with previously published data in limiting cases is performed and they are in excellent agreement.
\end{abstract}

Keywords: Carreau fluid model; nonlinear stretching sheet; nonlinear thermal radiation; convective boundary condition; non-uniform heat source/sink; numerical solution.

\section{INTRODUCTION}

${ }^{2}$ During the past four decades, the study of non-Newtonian fluids has gained interest because of their numerous technological applications, including manufacturing of plastic sheets, the performance of lubricants, and movement of biological fluids. In particular, the flow of an incompressible non-Newtonian fluid over a stretching sheet has several industrial applications in, for example, extrusion of a polymer sheet from a dye or in the drawing of plastic films. In view of their differences with Newtonian fluids, several models of non-Newtonian fluids have been proposed. Some materials, eg., melts, muds, condensed milk, glues, printing ink, emulsions, soaps, sugar solution, paints, shampoos, tomato paste, show the non-Newtonian properties of fluids. The governing equations of non-Newtonian fluids are highly non-linear and much more complicated compared to those of Newtonian fluids. Unlike power-law fluid, the Carreau model is one of the non-Newtonian fluid models for which constitutive relationship holds at both low and high shear rates. Due to this fact, it has achieved wider acceptance at present. Heat and mass transfer in the hydromagnetic flow of Carreau fluid past a vertical porous plate with thermal radiation and thermal diffusion are addressed by Olajuwon (2011). The three-dimensional peristaltic motion of Carreau fluid in a rectangular duct was described by Ellahi et al (2014). Riaz et al (2014) presented the peristaltic flow of a Carreau fluid in a non-uniform tube under the consideration of long wavelength. Hayat et al (2014) reported the two-dimensional boundary layer flow of Carreau fluid past a permeable stretching sheet with convective boundary condition. Akbar et al (2014) numerically carried out to study the two-dimensional stagnation-point flow of an in-compressible Carreau fluid toward a shrinking surface. Pantokratoras(2016) considered the flow of a nonNewtonian, Carreau fluid, directed normally to a horizontal, stationary, circular cylinder. Khan et al (2016) analyzed the impact of magnetic field on the stagnation-point flow of a generalized Newtonian Carreau fluid. By employing homotopy analysis method (HAM) Abolbashari et al(2014) study the entropy analysis in an unsteady magnetohydrodynamic nano-fluid regime adjacent to an accelerating stretching permeable surface with the water as the base fluid. Freidoonimehr et al (2015) have Investigated the transient MHD laminar free convection flow of nano-fluid past a vertical surface embedded in a porous and stretched under acceleration. They proved that selecting Aluminum oxide and Copper as the nanoparticle leads to the minimum and maximum amounts of skin friction coefficient absolute value, and also Copper and Titanium dioxide nanoparticles have the largest and lowest local Nusselt number. A recent articles related to the topic is cited in (Abolbashari et al.,2015; Rashidi et al.,2011).

In most of the above-mentioned papers, the physical situations were related to a linearly stretching sheet. But the stretching may not

*Corresponding Author. Email: dr.bcprasanna@gmail.com 
necessarily be linear; it may be quadratic, power-law, exponential, and so on. The flow and heat transfer caused by a nonlinearly stretching sheet is of paramount interest. Bataller (2008) obtained the similarity solutions for flow and heat transfer over a nonlinearly stretching sheet by considering both uniform and variable surface temperatures. Mukhopadhyay (2013) presented the boundary layer flow and heat transfer of non-Newtonian fluid over a nonlinear stretching sheet. Nandeppanavar et al. (2011) investigated the viscoelastic boundary layer flow and heat transfer over an exponentially stretching continuous sheet in the presence of a heat source/sink. Yazdi et al (2014) reported the steady slip boundary layer flow and heat transfer of a non-Newtonian power-law fluid over continuously nonlinear moving permeable surface at prescribed surface temperature including the effects of viscous dissipation and internal heat generation/absorption. Krishnamurthy et al (2016) examined the thermal radiation and chemical reaction effect on MHD velocity slip boundary layer flow and melting heat transfer of nanofluid induced by a nonlinear stretching heet. Raju et al (2016) analyzed the flow, heat and mass transfer behavior of Casson fluid past an exponentially permeable stretching surface in presence of thermal radiation, magnetic field, viscous dissipation, heat source and chemical reaction. Recently, Khan et al (2016) visualized the boundary layer flow and heat transfer to a Carreau model over a non-linear stretching surface considered the effect of nonlinear thermal radiation and convective boundary condition. Prasannakumara and Shashikumar (2017) studied MHD flow and heat transfer of nanofluid over a nonlinear stretching sheet combined with fluid particle suspension in the presence of thermal radiation.

Heat transfer in the laminar boundary layer flow over a stretching sheet influenced by thermal radiation has numerous industrial applications, including combustion, furnace design, nuclear reactor safety, fluidized bed heat exchangers, solar ponds, solar collectors, turbid water bodies, photochemical reactors and many others. Many processes in engineering areas occur at high temperature and knowledge of radiation heat transfer becomes very important for the design of the pertinent equipment. Nuclear power plants, gas turbines and the various propulsion devices for aircraft, missiles, satellites and space vehicles are examples of such engineering areas. It is difficult to construct a system in scientific and engineering applications in which the working fluids will have a small temperature difference. But in recent years, many authors studied the effect of nonlinear thermal radiation on heat transfer of both Newtonian and non-Newtonian fluids over stretching surfaces (Raju et.al.,2016a, Ramesh et al.,2017; Prasannakumara et al.,2017).

Motivated by the above literature and applications, the current investigation explores the boundary layer flow and heat transfer of Carreau fluid model in a porous medium. Additionally, nonlinear radiative heat transfer, viscous dissipation and non-uniform heat source/sink are studied along with convective boundary conditions. The governing boundary layer equations are transformed into ordinary differential equations using the local similarity variables. Numerical solutions are computed for the velocity and temperature fields with the help of Runge-Kutta-Fehlberg fourth-fifth order method along with shooting technique. The effects of different flow parameters on the flow fields are elucidated through graphs and tables.

\section{MATHEMATICAL FORMULATION}

We consider the problem of an incompressible two-dimensional flow of a generalized non- Newtonian fluid, namely Carreau fluid, due to a stretching sheet which coincides with the plane $y=0$. It is assumed that the flow being confined to $y>0$. The stretching sheet has uniform temperature $T_{w}$ with $T_{\infty}\left(T_{w}>T_{\infty}\right)$ as the ambient fluid temperature and it is moving with a non-linear velocity $U_{w}=b x^{m}$. The parameters $b$ and $m(>0)$ are positive real numbers relating to the stretching speed. The $x$ coordinate is taken along the moving surface and the $y$ coordinate is taken normal to it.
Keeping the above assumptions in mind, the governing equations of the problems are

$$
\frac{\partial u}{\partial x}+\frac{\partial v}{\partial y}=0
$$

$u \frac{\partial u}{\partial x}+v \frac{\partial u}{\partial y}=v \frac{\partial^{2} u}{\partial y^{2}}\left[1+\lambda^{2}\left(\frac{\partial u}{\partial y}\right)^{2}\right]^{\frac{(n-1)}{2}}+v(n-1) \lambda^{2}\left[\frac{\partial^{2} u}{\partial y^{2}}\left(\frac{\partial u}{\partial y}\right)^{2}\right]$

$\left[1+\lambda^{2}\left(\frac{\partial u}{\partial y}\right)^{2}\right]^{\frac{(n-3)}{2}}-\frac{v}{k^{\prime}} u$

$c_{p} \rho\left(u \frac{\partial T}{\partial x}+v \frac{\partial T}{\partial y}\right)=\alpha \frac{\partial^{2} T}{\partial y^{2}}-\frac{\partial q_{r}}{\partial y}+\mu\left(\frac{\partial u}{\partial y}\right)^{2}+q^{\prime \prime \prime}$,

The associated boundary conditions are:

$u=U_{W}=b x^{m} v=0,-k \frac{\partial T}{\partial y}=h_{f}\left(T_{f}-T\right)$ at $y=0$,

$u \rightarrow 0, T \rightarrow T_{\infty}$ as $y \rightarrow \infty$

Where $u$ and $v$ represent the velocity components in the $x$ and $y$ directions, respectively, $\alpha=\frac{k}{\rho c_{p}}, \alpha$ is the thermal diffusivity, $c_{p}$ is the specific heat and $k$ is the thermal conductivity, $h_{f}$ is the convective heat transfer coefficient.

Here, $q^{\prime \prime \prime}$ is the space and temperature dependent internal heat generation/absorption (nonuniform heat source/sink) which can be expressed as,

$q^{\prime \prime \prime}=\left(\frac{k U_{w}(x)}{x v}\right)\left[A_{1}\left(T_{f}-T_{\infty}\right) f^{\prime}(\eta)+B_{1}\left(T-T_{\infty}\right)\right]$

where $T_{f}$ and $T_{\infty}$ denote the temperature at the wall and at the large distance from the wall respectively. $A_{1}$ and $B_{1}$ are the parameters of space and temperature dependent internal heat generation/absorption. It is to be noted that $A_{1}$ and $B_{1}$ are positive to the internal heat source and negative to an internal heat sink.

Unlike the linearized Rosseland approximation, we use nonlinear Rosseland diffusion approximation from which one can obtain results for both small and large differences between $T_{f}$ and $T_{\infty}$.

Using Rosseland (Pantokratoras and Fang.,2013) approximation for radiation, the radiative heat flux is simplified as,

$q_{r}=-\frac{4 \sigma^{*}}{3 k^{*}} \frac{\partial T^{4}}{\partial y}=\left(-\frac{16 \sigma^{*} T^{3}}{3 k^{*}}\right) \frac{\partial T}{\partial y}$

where $\sigma^{*}$ is the Stefan-Boltzmann constant and $k^{*}$ is the mean absorption coefficient.

In view to Eq. (5) and (6), energy equation (3) will take the form

$c_{p} \rho\left(u \frac{\partial T}{\partial x}+v \frac{\partial T}{\partial y}\right)=\frac{\partial}{\partial y}\left[\left(k+\frac{16 \sigma^{*} T^{3}}{3 k^{*}}\right) \frac{\partial T}{\partial y}\right]+\mu\left(\frac{\partial u}{\partial y}\right)^{2}+q^{\prime \prime \prime}$,

The governing momentum and heat transfer equations can be transferred into the coupled ordinary differential equations by introducing the following suitable transformations:

$\psi(x, y)=\sqrt{\frac{2 v b}{m+1}} x^{\frac{m+1}{2}} b x f^{\prime}(\eta), \quad \eta=y \sqrt{\frac{(m+1) b}{2 v}} x^{\frac{m-1}{2}}$, 
with $T=T_{\infty}\left(1+\left(\theta_{w}-1\right) \theta\right)$, with $\theta_{w}=\frac{T_{f}}{T_{\infty}}, \theta_{w}>1$ being the temperature ratio parameter.

where $\psi$ is the stream function. Where $u=\frac{\partial \psi}{\partial y}$ and $v=-\frac{\partial \psi}{\partial x}$

The momentum and energy equations (2), (3), (6) and (10) yield

$\left(1+n W e^{2} f^{\prime \prime 2}\right)\left[1+W e^{2} f^{\prime 2}\right]^{\frac{n-3}{2}} f^{\prime \prime \prime}+f f^{\prime \prime}\left(\frac{2 m}{m+1}\right) f^{\prime 2}-k_{p} f^{\prime}=0$

$\left[1+N r\left(1+\left(\theta_{w}-1\right) \theta\right)^{3} \theta^{\prime}\right]^{\prime}+\operatorname{Pr} f \theta^{\prime}+\operatorname{Pr} E c\left(f^{\prime \prime}\right)^{2}+A_{1} f^{\prime}+B_{1} \theta=0$

The relevant boundary condition becomes:

$f^{\prime}(0)=1, f(0)=0, \theta^{\prime}(0)=-B i(1-\theta(0))$,

$f^{\prime}(\infty)=0, \theta(\infty)=0$,

where prime denotes differentiation with respect to $\eta$, $W e=\left(\frac{b^{3}(m+1) \Gamma^{2} x^{3 m-1}}{2 v}\right)^{\frac{1}{2}}$ the local Weissenberg number, $N r=\frac{16 \sigma^{*} T_{\infty}^{3}}{3 k k^{*}}$ is the radiation parameter, $\theta_{w}$ is the temperature ratio parameter, $\operatorname{Pr}=\frac{\mu c_{p}}{\rho}$ is the Prandtl number, $E c=\frac{U_{w}^{2}}{\left(T_{f}-T_{\infty}\right) c_{p f}}$ is the Eckert number, $B i=-\frac{h_{f}}{k_{f}} \operatorname{Re}_{x}^{-\frac{1}{2}}$ is the Biot number and $k_{p}=\frac{v}{k^{\prime} b}$ is the permeability parameter.

It is to be noted that for $n=1$ or $W e=0$ the Carreau fluid transform to the Newtonian fluid.

The dimensionless physical quantities of practical concern are the local skin friction coefficient $C_{f x}$ and the local Nusselt number $N u_{x}$, which are given by

$N u_{x}=\frac{x q_{w}}{k\left(T_{f}-T_{\infty}\right)} \quad$ and $\quad C_{f}=\frac{\tau_{w}}{\rho U_{w}^{2}(x)}$,

where the shear stress $\left(\tau_{w}\right)$ and surface heat flux $\left(q_{w}\right)$ given by

$\tau_{w}=n_{0} \frac{\partial u}{\partial y}\left(1+\Gamma^{2}\left(\frac{\partial u}{\partial y}\right)^{2}\right)^{\frac{n-1}{2}}, \quad q_{w}=-k \frac{\partial T}{\partial y}+\left(q_{r}\right)_{w}$,

Using the non-dimensional variables, we obtain

$\sqrt{\operatorname{Re}_{x}} C_{f}=f^{\prime \prime}(0) \sqrt{\frac{m+1}{2}}\left[1+W e^{2} f^{\prime \prime}(0)^{2}\right]^{\frac{n-1}{2}}$,

$N u_{x}=-\sqrt{\frac{m+1}{2}} \operatorname{Re}_{x}^{\frac{1}{2}}\left(1+N r \theta^{3}{ }_{w}\right) \theta^{\prime}(0)$,

where $\operatorname{Re}_{x}=\frac{U^{2} w}{b v}$ is the local Reynold's number.

\section{NUMERICAL METHOD}

The resultant ordinary differential equations are coupled and nonlinear in nature, therefore, a closed-form solution may not be possible. Thus, the RKF-45 method with the shooting technique is used in the present study to solve the boundary-layer equations (9)-(10) when subjected to the boundary conditions equations (11) and (12). The shooting technique is an iterative algorithm that attempts to identify appropriate initial conditions for a relevant initial value problem that provides the solution to the original boundary value problem. This algorithm is proven to be precise and accurate, and it has been successfully used to solve a wide range of nonlinear problems in transport phenomena, especially flow and heat transfer problems. The equations are first reduced into system of first order simultaneous equations. Missing initial conditions are accessed with the help of Shooting technique. In addition, the relative error tolerance to $10^{-6}$ is considered for convergence and the step size is chosen as $\Delta \eta=0.001$. The CPU running-time for existing numerical solution is $0.033 \mathrm{sec}$. The asymptotic boundary conditions at $\eta_{\infty}$ are replaced by $\eta_{6}$, in accordance with $f^{\prime}\left(\eta_{\max }\right)=f^{\prime \prime}\left(\eta_{\max }\right)=\theta\left(\eta_{\max }\right)=0$ where $\eta_{\max }$ is a sufficiently large value of $\eta$ at which the boundary conditions Eq. (2.18) are asymptotically satisfied.

The accuracy of the aforementioned numerical method was validated by direct comparisons with the numerical results corresponding to the values of skin friction coefficient $\left[-f^{\prime \prime}(0)\right]$ with those reported by Cortell (2007), Hamad and Ferdows (2012) and Khan and Hashim (2015) for different values of stretching parameter $(m)$ by keeping $n=1$ and $k_{p}=0$. Further, we have made a comparison of values of heat transfer coefficient $\left[-\theta^{\prime}(0)\right]$ reported earlier by Cortell (2007) and Khan and Hashim (2015) for various values of $m$ in the absence of the $k_{p}=E c=\theta_{w}=0$ and $\operatorname{Pr}=1$ and they are presented in Tables 1 and 2 respectively. It can be seen from these tables that an excellent agreement between the results exists. This favorable comparison lends confidence in the numerical results to be reported in the next section. The values of skin friction coefficient and Nusselt number for the different value of the pertinent parameters in the presence and absence of stretching parameter and temperature ratio parameter was depicted in Table 3 and 4 respectively.

Table 1 A Comparison of the values of $-f^{\prime \prime}(0)$ with $n=1.0, W e=0.0, k_{p}=0$ for different values of $m$.

\begin{tabular}{ccccc}
\hline \hline$m$ & $\begin{array}{c}\text { Cortell } \\
(2007)\end{array}$ & $\begin{array}{c}\text { Hamad and } \\
\text { Ferdows } \\
(2012)\end{array}$ & $\begin{array}{c}\text { Khan and } \\
\text { Hashim } \\
(2015)\end{array}$ & $\begin{array}{c}\text { Present } \\
\text { result }\end{array}$ \\
\hline \hline 0.0 & 0.627547 & 0.6369 & 0.627554 & 0.627569 \\
1.0 & 1.0 & 1.0043 & 1.0 & 1.0000 \\
1.5 & 1.061587 & & 1.061601 & 1.061598 \\
3.0 & 1.148588 & 1.1481 & 1.148593 & 1.147682 \\
7.0 & 1.216847 & & 1.216851 & 1.216641 \\
10.0 & 1.234875 & 1.2342 & 1.234875 & 1.233676 \\
20.0 & 1.257418 & 1.2574 & 1.257424 & 1.257312 \\
\hline \hline
\end{tabular}

Table 2 A Comparison of the values of $-\theta^{\prime}(0)$ with $n=1.0, W e=0.0, K_{p}=0, N r=0, \theta_{w}=0, E c=0$ and $\operatorname{Pr}=1.0$, for different values of $m$.

\begin{tabular}{cccc}
\hline \hline$m$ & Cortell (2007) & $\begin{array}{c}\text { Khan and } \\
\text { Hashim (2015) }\end{array}$ & Present study \\
\hline \hline 0.2 & 0.610262 & 0.610202 & 0.610242 \\
0.5 & 0.595277 & 0.595201 & 0.595265 \\
1.5 & 0.574537 & 0.574730 & 0.574625 \\
3.0 & 0.564472 & 0.564662 & 0.564562 \\
10 & 0.554960 & 0.554951 & 0.554895 \\
\hline \hline
\end{tabular}

\section{RESULT AND DISCUSSION}

The effect of nonlinear thermal radiation on boundary layer flow and heat transfer of Carreau fluid model over a nonlinear stretching sheet embedded in a porous medium in the presence of non-uniform heat source/sink and viscous dissipation with convective boundary condition have been studied. The purpose of this section is to analyze the effects of various physical parameters such as permeability parameter $\left(k_{p}\right)$, Weissenberg number $(W e)$, power law index $(n)$, stretching parameter 
$(m)$, radiation parameter $(N r)$, temperature ratio parameter $\left(\theta_{w}\right)$, Biot number $\left(B_{i}\right)$, Eckert number $(E c)$, Prandtl number $(\operatorname{Pr})$ and heat source/sink parameters $\left(A_{1} \& B_{1}\right)$, on the velocities and temperature fields as well as skin friction coefficient and local Nusselt number profiles. Therefore, for such objective, Figs.1-15 has been plotted. In numerical computations throughout we employed,

$k_{p}=0.3, W e=0.5, n=2, m=1.5, A_{1}=B_{1}=0.5, \theta_{w}=1.2, N r=1.5, B_{i}=2$ and $\operatorname{Pr}=6.2$.

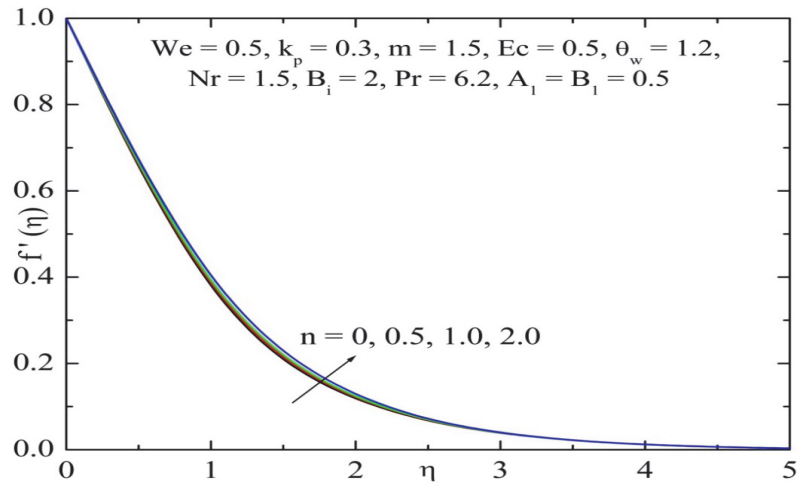

Fig. 1 Velocity profile $f^{\prime}(\eta)$ for several values of power law index $n$.

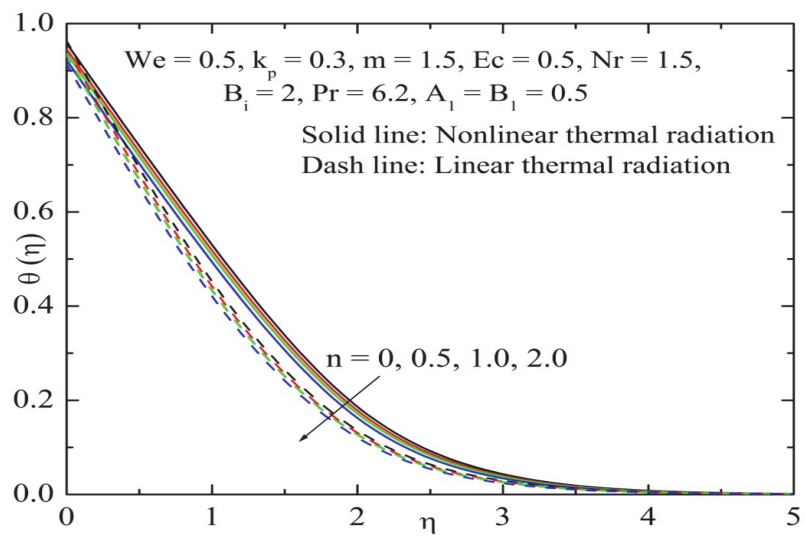

Fig. 2 Temperature profile $\theta(\eta)$ for several values of power law index

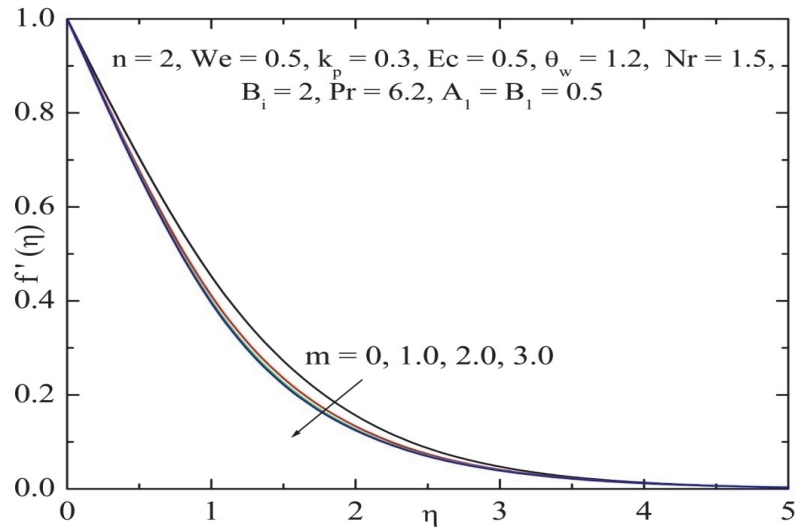

Fig. 3 Velocity profile $f^{\prime}(\eta)$ for several values of the stretching parameter $m$.
Figures 1 and 2 shows the effect of power law index $(n)$ on velocity and temperature profiles respectively. The velocity profile represents an increasing behavior for increasing values of $n$ as shown in figure 1 . Figure 2 explains that the temperature profile decreases with increasing power law index $(n)$. Further this figure reveals that there is a corresponding momentum boundary layer thickness decreases by increasing the power law index $(n)$

Figures 3 and 4 characterize the velocity and temperature profiles for distinct values of the stretching parameter $(m)$. Figure 3 indicates that fluid velocity decreases with the increasing values of the $\mathrm{m}$. From figure 4 , it is noticeable that the influence of the stretching parameter $(m)$ is to increase the temperature profile.

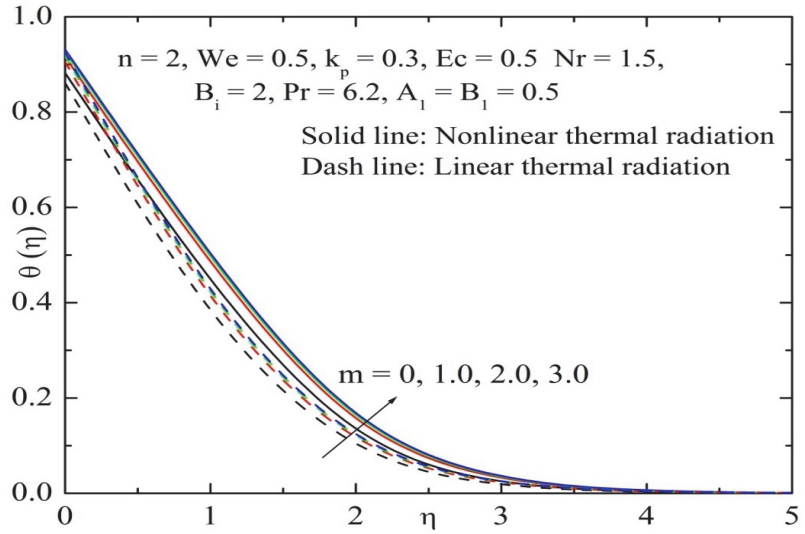

Fig. 4 Temperature profile $\theta(\eta)$ for several values of the stretching parameter $m$.

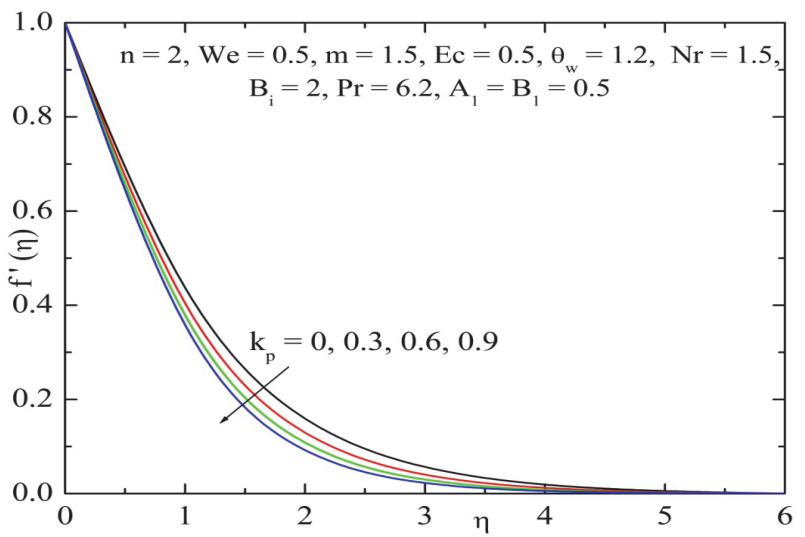

Fig. 5 Velocity profile $f^{\prime}(\eta)$ for several values of the permeability parameter $k_{n}$.

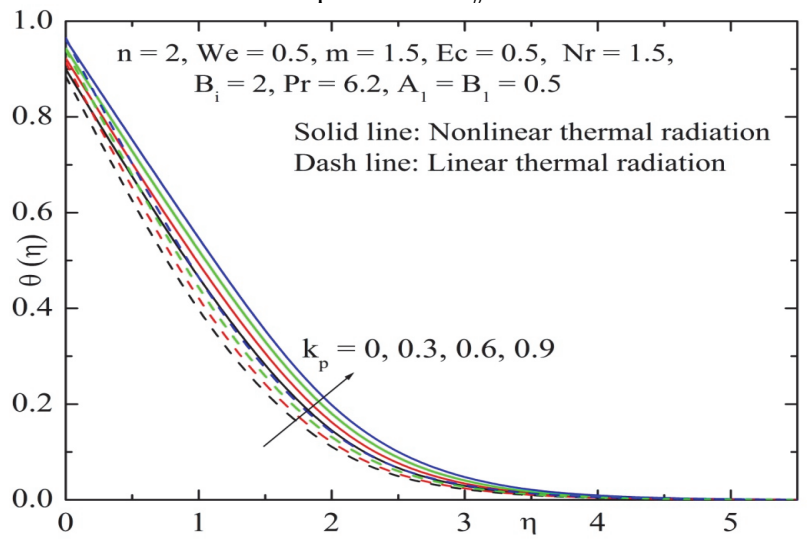

Fig. 6 Temperature profile $\theta(\eta)$ for several values of the permeability parameter $k_{p}$. 
The influence of porous parameter $\left(k_{p}\right)$ on velocity and temperature profiles as drawn in figures 5 and 6 respectively. Figure 5 elucidates that, the velocity field and momentum boundary layer thickness reduces by increasing porous parameter. Further, the temperature profile enhanced with porous parameter as shown in Figures 6. Physically speaking the boundary layer thickness increases by increasing the porous parameter.

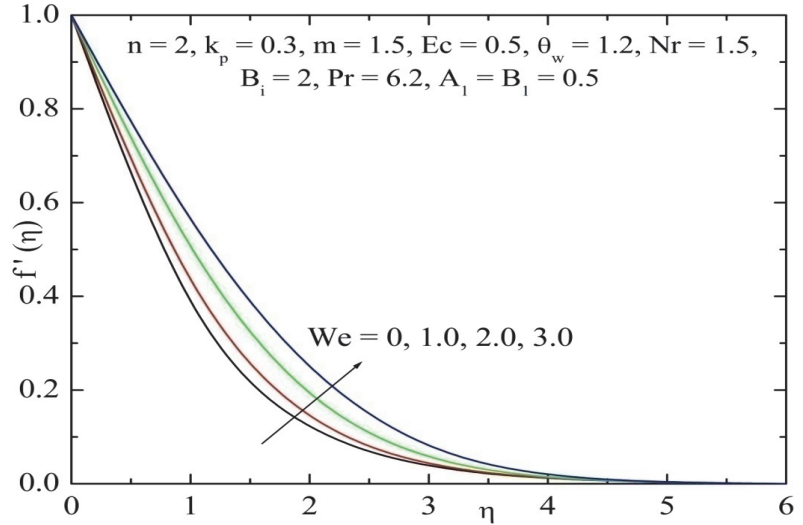

Fig. 7 Velocity profile $f^{\prime}(\eta)$ for several values of the Weissenberg number $W e$.

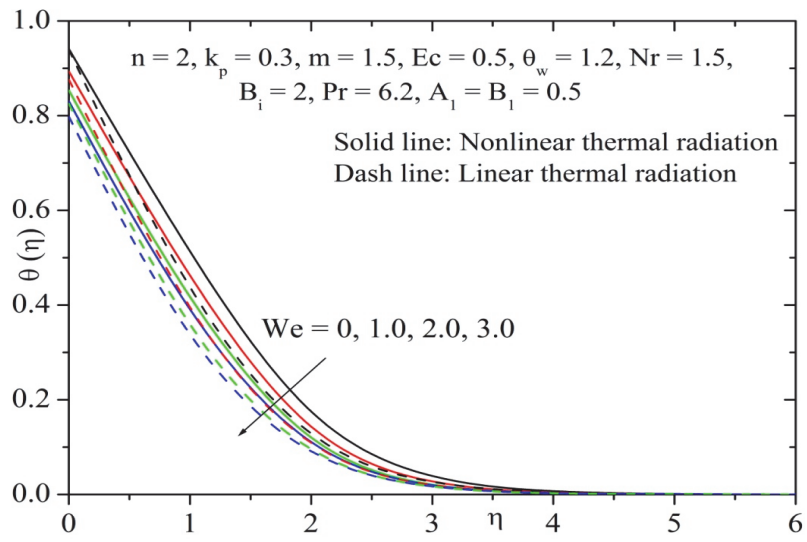

Fig. 8 Temperature profile $\theta(\eta)$ for several values of the Weissenberg number $W e$.

Figures 7 and 8 emblematize the effects of the Weissenberg number $W e$ on the velocity and temperature profile respectively. Figure 7 examines that the velocity profile and boundary layer thickness increases by uplifting values of Weissenberg number. On the other hand influence of the Weissenberg number on the temperature distribution is represent in figure 8 and it is clear that the temperature distribution decreases by increasing the Weissenberg number.

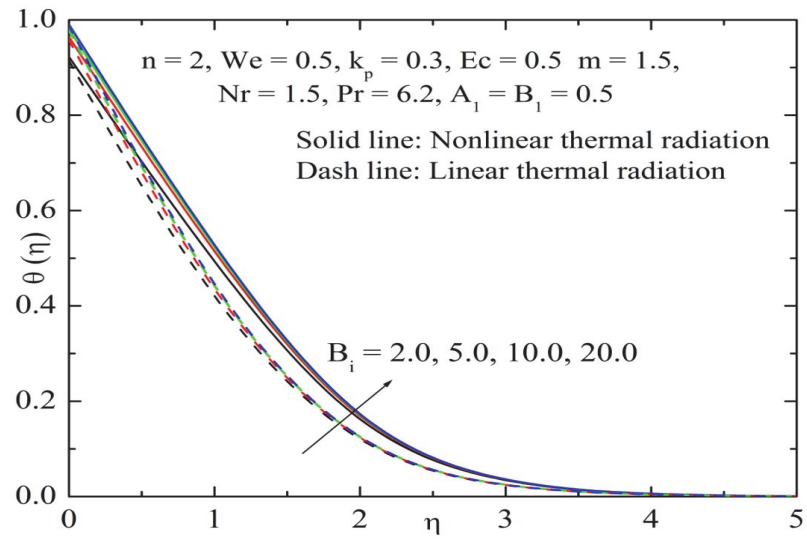

Fig. 9 Temperature profile $\theta(\eta)$ for several values of the Biot number $B_{i}$.
Figure 9 dissemble the change in temperature profile corresponding to different values of Biot number $\left(B_{i}\right)$. We have seen that the extent values of Biot number lead to elevated temperature and thicker thermal boundary layer thickness. Biot number $\left(B_{i}\right)$ depends on heat transfer coefficient $h_{1}$ which is stronger for larger Biot number that shows higher temperature.

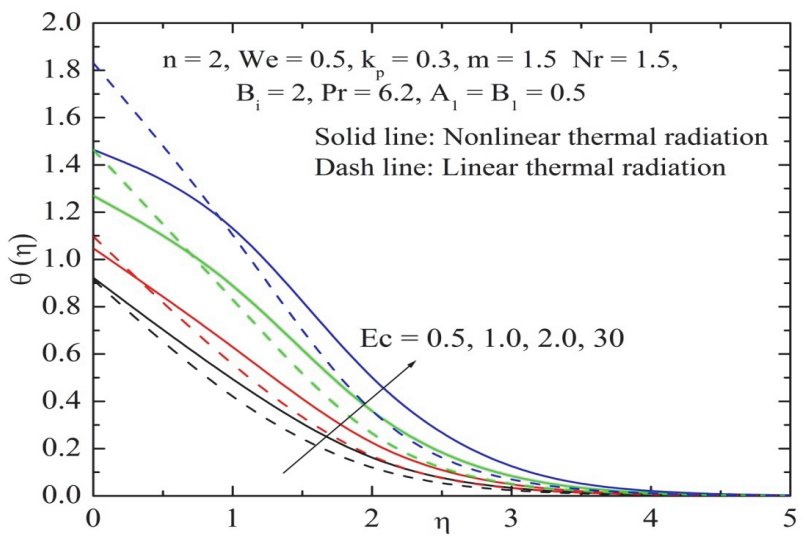

Fig. 10 Temperature profile $\theta(\eta)$ for several values of the Biot number $B_{i}$.

Figure 10 is sketched the temperature distributions against the Eckert number $(E c)$. The effect of increasing values of Eckert number is to increase temperature distribution. This is due to the fact that, increasing in the values of Eckert number enhances the kinetic energy and this leads to the increases in the temperature and thermal boundary layer thickness.

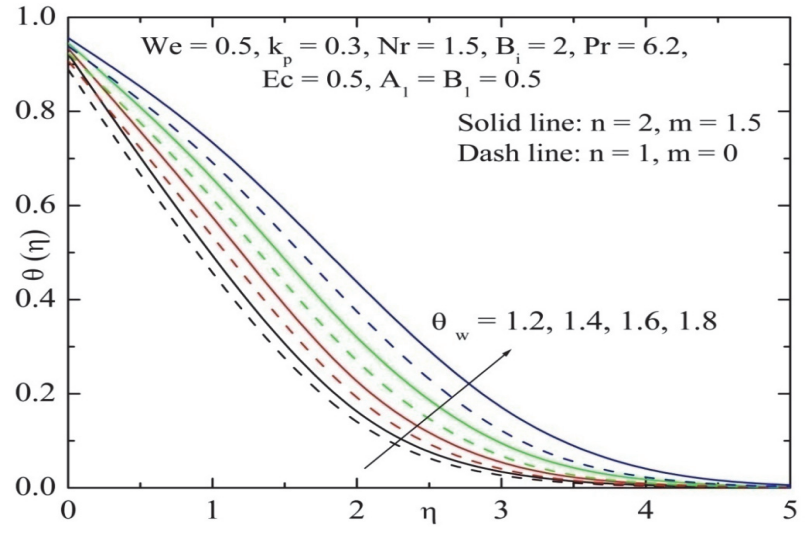

Fig. 11 Temperature profile $\theta(\eta)$ for several values of the temperature ratio parameter $\theta_{w}$

The effect of temperature ratio parameter over the dimensionless temperature $\left(\theta_{w}\right)$ is shown in figure 11. Increasing $\theta_{w}$ surface temperature parameter is to increase the temperature. Figure 12 illustrates the effects of radiation parameter $(\mathrm{Nr})$ over the temperature profiles. It is observed that the effect radiation parameter is to reduce the temperature, elucidating the face that the thermal boundary layer thickness decreases as $\mathrm{Nr}$ increases.

The variation of temperature distributions for various values of space dependent heat source/sink parameter $A_{1}$ and temperaturedependent heat source/sink parameter $B_{1}$ are represented graphically as shown in Figure 13-14. The heat source-sink parameter $A_{1}$ and $B_{1}$ are increasing, the boundary layer thickness also increases in both parameters profiles. 


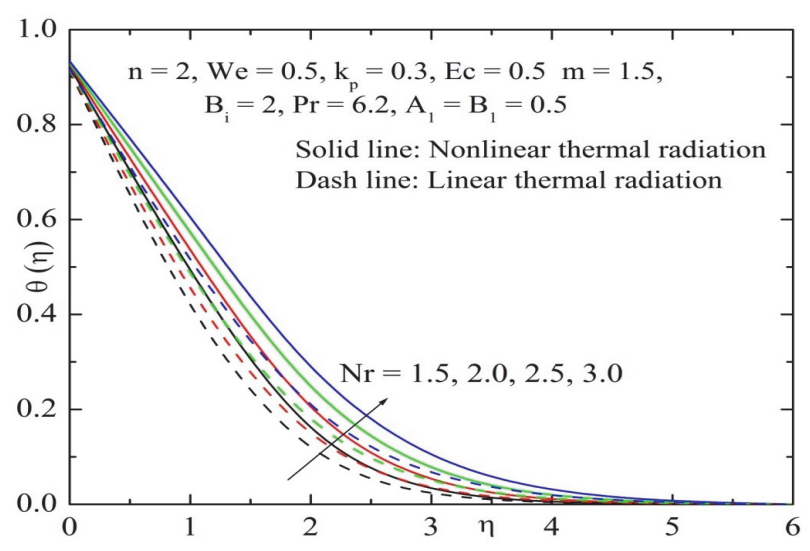

Fig. 12 Temperature profile $\theta(\eta)$ for several values of the radiation parameter $\theta_{w}$.

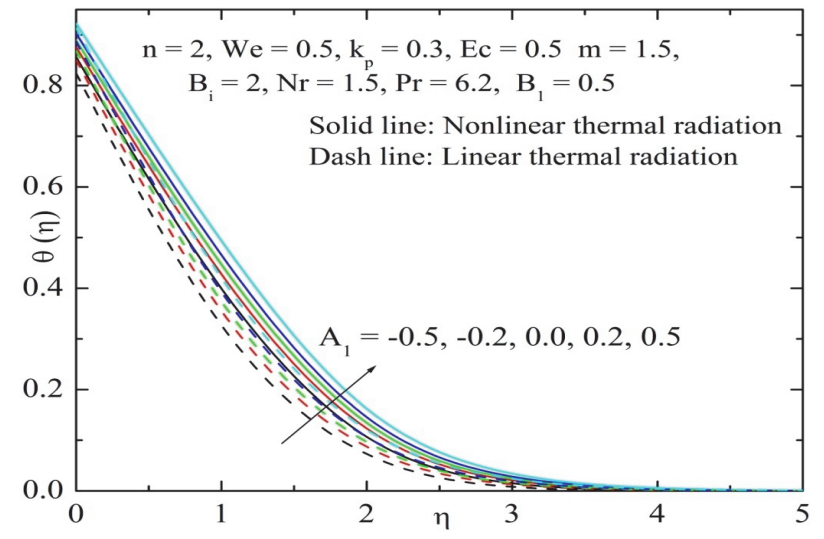

Fig. 13 Temperature profile $\theta(\eta)$ for several values of the space dependent $A_{1}$.

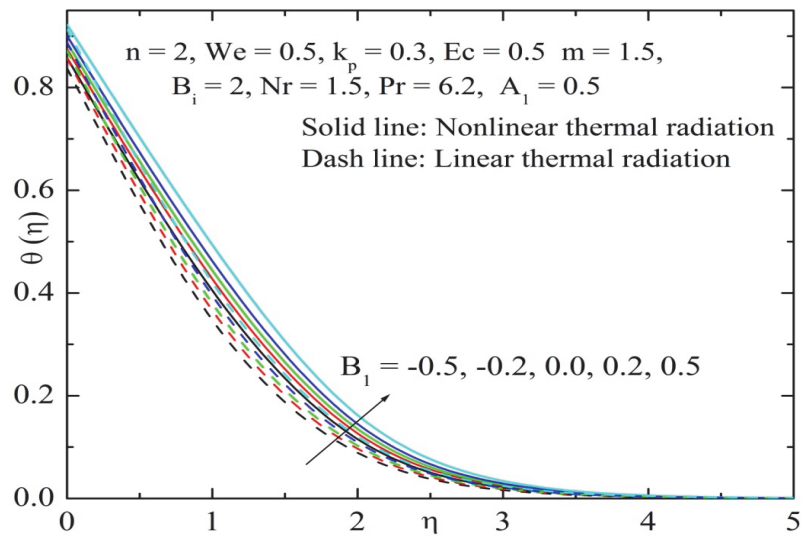

Fig. 14 Temperature profile $\theta(\eta)$ for several values of the temperature dependent $B_{1}$.

Figure 15 depicts the effect of Weissenberg number with permeability parameter on Skin friction coefficient. It can be concluded that Skin friction coefficient increases with permeability parameter and decreases for increasing values of Weissenberg number in both the cases.

Values of skin friction coefficient for different values of the pertinent parameter for both linear and nonlinear stretching casess are given in table 3 . From this table we can see that, skin friction coefficient values for all the considered parameter are high on nonlinear stretching surface compared to linear stretching.

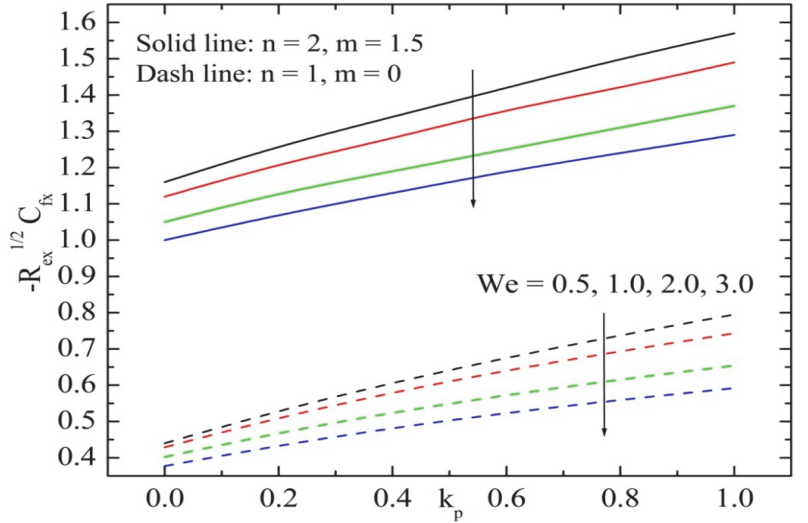

Fig. 15 Skin friction coefficient for several values of the Weissenberg number $W e$ with permeability parameter $k_{p}$.

Table 3 Values of skin friction coefficient for different values of the pertinent parameters.

\begin{tabular}{rrrr}
\hline \hline & & $\begin{array}{c}\text { Linear stretching } \\
\text { sheet: } n=1, m=0\end{array}$ & $\begin{array}{r}\text { Nonlinear stretching } \\
\text { sheet: } n=3, m=1.5\end{array}$ \\
\hline \hline$k_{p}$ & $W e$ & $-\sqrt{R_{e x}} C_{f x}$ & $-\sqrt{R_{e x}} C_{f x}$ \\
\hline \hline 0 & 0.5 & 0.4400 & 2.6823 \\
0.5 & 0.5 & 0.6425 & 3.6174 \\
1 & 0.5 & 0.7945 & 4.4830 \\
0.3 & 0 & 0.5796 & 1.3364 \\
0.3 & 0.3 & 0.5758 & 1.3245 \\
0.3 & 0.6 & 0.5657 & 1.3019 \\
\hline \hline
\end{tabular}

\section{CONCLUSIONS}

In this study, we have obtained the boundary layer equations of motion for a two-dimensional flow and nonlinear radiative heat transfer of an incompressible non-Newtonian Carreau fluid in the presence of nonuniform heat source/sink and viscous dissipation with convective boundary condition. The suitable transformations were employed to reduce the non-linear equations into self-similar ordinary differential equations. The Runge-Kutta-Fehlberg fourth-fifth order method along with shooting technique was used to solve the problem. The results were presented graphically and the effects of the various emerging parameters were discussed. The major findings from the present study are as follows:

- An increase in the momentum boundary layer thickness and a decrease in thermal boundary layer thickness was observed for the increasing values of the power law index $(n)$

- $\quad$ Role of the increasing values of the stretching parameter $(m)$ was to decrease the momentum boundary layer however, the opposite trend was noted for thermal boundary layer

- Velocity profile decreases and temperature profile increases for permeability parameter $\left(k_{p}\right)$.

- Increasing the Weissenberg number reduces the velocity profile and increases the temperature profiles.

- The effect of space and temperature dependent heat source/sink parameters $A_{1}$ and $B_{1}$ is to generate temperature for increasing positive values and absorb temperature for decreasing negative values. Hence, space and temperature dependent heat sinks are better suited for cooling purposes.

- Eckert number $(E c)$ temperature ratio $\left(\theta_{w}\right)$ and radiation parameter $(\mathrm{Nr})$ increases the thermal boundary layer thickness. 
- The temperature and thermal boundary layer thickness were depressed by increasing the Prandtl number (Pr) .

- Skin friction coefficient decreases for increasing the values of We with $k_{p}$.

\section{ACKNOWLEDGEMENT}

This research is supported by the University Grants Commission, New Delhi, India under the Major research project Scheme [F. No-43419/2014(SR)].

\section{REFERENCES}

Akbar, N.S., Nadeem, S., Rizwan U1 Haq., and Shiwei Ye., 2014, "MHD Stagnation Point flow of Carreau Fluid toward a Permeable Shrinking Sheet: Dual Solutions", Ain Shams Engineering Journal, 5, 1233-1239. https://doi.org/10.1016/j.asej.2014.05.006

Arshad Riaz, S.,Nadeem, R., Akbar, E., 2014, "Series Solution of Unsteady Peristaltic Flow of a Carreau Fluid in Small Intestines", Int. J. Biomath, 7(5), 1450049,1-9.

http://dx.doi.org/10.1142/S1793524514500491

Bataller, R.C., 2008, "Similarity Solutions for Flow and Heat Transfer of a Quiescent Fluid over a Non-Linearly Stretching Surface". Journal of Materials Processing Technology, 203, (1-3) 176-183.

https://doi.org/10.1016/j.jmatprotec.2007.09.055

Cortell, R., 2007, "Viscous Flow and Heat Transfer Over A Nonlinearly Stretching Sheet," Appl. Math. Comput..184(2), 864-873.

https://doi.org/10.1016/j.amc.2006.06.077

Ellahi, R., Bhatti, M.M., Riaz, A., and Sheikholeslami, M., 2014, "Effects of Magneto Hydrodynamics on Peristaltic Flow of Jeffrey Fluid in A Rectangular Duct Through a Porous Medium", Journal of Porous Media, 17(2), 143-157.

DOI: 10.1615/JPorMedia.v17.i2.50

Hamad, M.A.A., and Ferdows, M., 2012, "Similarity Solutions to Viscous Flow and Heat Transfer of Nanofluid Over Nonlinearly Stretching Sheet," Appl. Math. Mech. Eng., 33(7), 923-930.

DOI 10.1007/s10483-012-1595-7

Hayat, T.,Asad,S.,Mustafa, M., and Alsaedi, A., 2014, "Boundary Layer Flow of Carreau Fluid Over a Convectively Heated Stretching Sheet", Applied Mathematics and Computation, 246, 12-22.

https://doi.org/10.1016/j.amc.2014.07.083

Khan, M., and Hashim., 2015, "Boundary Layer Flow and Heat Transfer to Carreau Fluid Over a Nonlinear Stretching Sheet", AIP ADVANCES, 5, 107203,1-14.

http://dx.doi.org/10.1063/1.4932627

Khan, M., Hashim., Hussain, M., Azam, M., 2016, "Magnetohydrodynamic Flow of Carreau Fluid Over a Convectively Heated Surface in the Presence of Nonlinear Radiation", Journal of Magnetism and Magnetic Materials, 412, 63-68. https://doi.org/10.1016/i.jmmm.2016.03.077

Krishnamurthy, M.R., Gireesha, B.J., Prasannakumara, B.C., Gorla, R.S.R., 2016, "Thermal Radiation and Chemical Reaction Effects on Boundary Layer Slip Flow and Melting Heat Transfer of Nanofluid Induced by a Nonlinear Stretching Sheet", Nonlinear Engineering, 5(3), 147-159.

https://doi.org/10.1515/nleng-2016-0013
Mohammad Hossein Abolbashari., Navid Freidoonimehr., Foad Nazari., Rashidi, M.M., 2014, "Entropy Analysis for an Unsteady MHD Flow Past a Stretching Permeable Surface in Nanofluid", Powder Technology, 267, 256-267.

https://doi.org/10.1016/j.powtec.2014.07.028

Mohammad Hossein Abolbashari., Navid Freidoonimehr., Foad Nazari., Mohammad Mehdi Rashidi., 2015, “Analytical Modeling of Entropy Generation for Casson nano-Fluid Flow Induced by a Stretching Surface”, Advanced Powder Technology, 26 (2), 542-552.

https://doi.org/10.1016/j.apt.2015.01.003

Mohammad Mehdi Rashidi., Eemaeel Erfani., 2011, “The Modified Differential Transform Method for Investigating Nano Boundary-Layers Over Stretching Surfaces", International Journal of Numerical Methods for Heat \& Fluid Flow, 21( 7), 864-883.

https://doi.org/10.1108/09615531111162837

Mukhopadhyay, S., 2013, "Casson Fluid Flow and Heat Transfer Over a Nonlinearly Stretching Surface”, Chin. Phys. B., 22(7), 074701. http://dx.doi.org/10.1088/1674-1056/22/7/074701

Nandeppanavar, M.M., Vajravelu, K., Subhas Abel, M., and Chiu-On Ng., 2011,"Heat Transfer Over a Nonlinearly Stretching Sheet with NonUniform Heat Source and Variable Wall Temperature", International Journal of Heat and Mass Transfer, 54(23-24), 4960-4965.

https://doi.org/10.1016/j.ijheatmasstransfer.2011.07.009

Navid Freidoonimehr., Mohammad Mehdi Rashidi.,_Shohel Mahmud., 2015, "Unsteady MHD free Convective Flow Past a Permeable Stretching Vertical Surface in a Nano-Fluid", 87, 136-145.

https://doi.org/10.1016/j.ijthermalsci.2014.08.009

Olajuwon, B.I., 2011, "Convection Heat and Mass Transfer in a Hydromagnetic Carreau Fluid Past a Vertical Porous Plate in the Presence of Thermal Radiation and Thermal Diffusion", Thermal Science, 15(2), 241-252.

DOI: $10.2298 /$ TSCI101026060O

Pantokratoras, A., 2016, "Steady Flow of a Non-Newtonian Carreau Fluid Across an Unconfined Circular Cylinder", Meccanica, 51(4), 1007-1016.

https://doi.org/10.1007/s11012-015-0258-1

Pantokratoras, A., and Fang, T., 2013, "Sakiadis Flow with Nonlinear Rosseland Thermal Radiation", Physic Scrip., 87(1), 1-5.

https://doi.org/10.1088/0031-8949/87/01/015703

Prasannakumara, B.C., Gireesha, B.J., Gorla, R.S.R., and Krishnamurthy, M.R., 2016, "Effects of Chemical Reaction and Nonlinear Thermal Radiation on Williamson Nanofluid Slip Flow Over a Stretching Sheet Embedded in a Porous Medium",Journal of Aerospace Engineering 29 (5), 04016019,1-10.

https://doi.org/10.1061/(ASCE)AS.1943-5525.0000578

Prasannakumara, B.C., Shashikumar, N.S., 2017,"Boundary Layer Flow and Heat Transfer of Nanofluid with Fluid Particle Suspension Over a Nonlinear Stretching Sheet in the Presence of Thermal Radiation", Journal of Nanofluids, 6(3), 487-495.

http://dx.doi.org/10.1166/jon.2017.1346

Raju, C.S.K., Sandeep, N., Gnaneswara Reddy, M., 2016a "Effect of Nonlinear Thermal Radiation on 3D Jeffrey Fluid Flow in the Presence 
of Homogeneous-Heterogeneous Reactions", International Journal of Engineering Research in Africa, 21, 52-68.

http://dx.doi.org/10.4028/www.scientific.net/JERA.21.52

Raju, C.S.K., Sandeep, N., Sugunamma, V., Jayachandra Babu., M., Ramana Reddy, J.V., 2016a, "Heat and Mass Transfer in Magnetohydrodynamic Casson Fluid Over an Exponentially permeable Stretching Surface",Engineering Science and Technology, an International Journal,19(1),45-52.

https://doi.org/10.1016/j.jestch.2015.05.010
Ramesh, G.K., Prasannakumara, B.C., Gireesha, B.J., Shehzad, S.A., Abbasi, F.M., 2017, "Three Dimensional Flow of Maxwell Fluid with Suspended Nanoparticles Past a Bidirectional Porous Stretching Surface with Thermal Radiation", Thermal Science and Engineering Progress, 1,6-14.

https://doi.org/10.1016/j.tsep.2017.02.006

Yazdi, M.H., Hashim, I., Moey, L. K., Sopian, K., 2014, “Slip Velocity Effect on Non-Newtonian Fluid Flow Over Moving Permeable Surface with Nonlinear Velocity and Internal Heat Generation/Absorption", International Review on Modelling and simulations, 7(4), 661-670. http://dx.doi.org/10.15866/iremos.v7i4.1004 